\title{
An infinite family of totally real number fields
}

\author{
by
}

\section{Humio Ichimura (Yokohama) and Fuminori Kawamoto (Tokyo)}

1. Introduction. This is a continuation of [7]. Let $F$ be a totally real number field of degree $n(\geq 2)$ and $\iota_{i}(1 \leq i \leq n)$ all real embeddings of $F$. We denote by $\mathfrak{l}_{i}$ the real prime of $F$ corresponding to $\iota_{i}$ and put $\mathfrak{l}_{0}=\mathfrak{l}_{0}(F):=\mathfrak{l}_{1} \mathfrak{l}_{2} \ldots \mathfrak{l}_{n}$. For $\mathfrak{l} \mid \mathfrak{l}_{0}, F(\mathfrak{l})$ denotes the ray class field of $F \bmod \mathfrak{l}$. In particular, $F(1)$ is the Hilbert class field of $F$. Let $K / F$ be a subextension of $F(\mathfrak{l}) / F$ and $G$ its Galois group. We denote by $\mathfrak{o}_{F}$ and $\mathfrak{o}_{K}$ the rings of integers in $F$ and $K$, respectively. If there exists some $x$ in $\mathfrak{o}_{K}$ such that $\{s(x)\}_{s \in G}$ is a free $\mathfrak{o}_{F}$-basis of $\mathfrak{o}_{K}$, then we say that the tamely ramified abelian extension $K / F$ has a normal integral basis (abbreviated NIB). Such an element $x$ is called a generator of NIB of $K / F$. We ask whether $K / F$ has an NIB. For this, we consider a subgroup of an elementary abelian 2-group $\mathfrak{o}_{F}^{\times} / \mathfrak{o}_{F}^{\times 2}$ :

$$
\begin{aligned}
\mathcal{N}^{\mathfrak{l}} & =\mathcal{N}^{\mathfrak{l}}(F) \\
& :=\left\{[\eta] \in \mathfrak{o}_{F}^{\times} / \mathfrak{o}_{F}^{\times 2} \mid \eta \in \mathfrak{o}_{F}^{\times}, \eta \equiv 1 \bmod 4, \iota_{i}(\eta)>0 \text { for all } \mathfrak{l}_{i} \mid \mathfrak{l}_{0} \mathfrak{l}^{-1}\right\} .
\end{aligned}
$$

Here, for a ring $R, R^{\times}$denotes the group of units in $R$ and $[\eta]$ is the residue class of $\eta$. We denote by $\mathbb{Z}$ the ring of all rational integers and put $\mathbb{F}_{2}:=$ $\mathbb{Z} / 2 \mathbb{Z}$. Then we can regard $\mathcal{N}^{\mathfrak{l}}$ as a vector space over $\mathbb{F}_{2}$ with dimension $\leq n$. Furthermore, we put

$$
L^{\mathfrak{l}}:=F\left(\left\{\sqrt{\eta} \mid[\eta] \in \mathcal{N}^{\mathfrak{l}}\right\}\right) .
$$

By Kummer theory, $L^{\mathfrak{l}}$ is a subfield of $F(\mathfrak{l})$ and $L^{\mathfrak{l}} / F$ is an elementary abelian 2-extension of degree $2^{\operatorname{dim} \mathcal{N}^{\prime}}$, where $\operatorname{dim} V$ is the dimension of an $\mathbb{F}_{2^{-}}$ vector space $V$. In [7], we proved the following theorem, using Brinkhuis [2, Corollary 2.10] (or [3, Corollary 2.1]) and Childs [4, Theorem B].

THEOREM 1. Let $F$ be a totally real number field and $\mathfrak{l} \mid \mathfrak{l}_{0}$.

(i) The extension $L^{\mathfrak{l}} / F$ is the maximal subextension of $F(\mathfrak{l}) / F$ which has an NIB. Furthermore, if $\left\{\left[\eta_{1}\right], \ldots,\left[\eta_{r}\right]\right\}$ is an $\mathbb{F}_{2}$-basis of $\mathcal{N}^{\mathfrak{l}}$ with $\eta_{i} \equiv$ $1 \bmod 4$, then $x:=\prod_{i=1}^{r}\left(\left(1+\sqrt{\eta_{i}}\right) / 2\right)$ is a generator of $N I B$ of $L^{\mathrm{l}} / F$. 
(ii) Let $K / F$ be a subextension of $F(\mathfrak{l}) / F$. Then $K / F$ has an NIB if and only if $K \subset L^{\mathfrak{l}}$. If the condition is satisfied, then $\operatorname{Tr}_{L^{\mathfrak{l}} / K}(x)$ is a generator of NIB of $K / F$. In particular, $F(\mathfrak{l}) / F$ has an NIB if and only if $h_{F}=$ $\left|\mathcal{N}^{\mathfrak{l}}\right|[F(\mathfrak{l}): F(1)]^{-1}$. Here, $\operatorname{Tr}_{L^{\mathfrak{l}} / K}$ is the trace map from $L^{\mathfrak{l}}$ to $K$ and $h_{F}$ denotes the class number of $F$.

In view of this theorem, the $\mathbb{F}_{2}$-vector space $\mathcal{N}^{\mathfrak{l}}$ is naturally of interest. In [7], we determined an $\mathbb{F}_{2}$-basis of $\mathcal{N}^{\mathfrak{l}}$ for all real quadratic fields and all cyclic cubic fields. The main purpose of this article is to determine an $\mathbb{F}_{2}$-basis of $\mathcal{N}^{\mathfrak{l}}$ and a generator $x$ of NIB of the abelian extension $L^{\mathfrak{l}} / F$ for a certain family of totally real number fields $F$ which are defined by Eisenstein polynomials

$$
f(X)=\prod_{i=1}^{n}\left(X-a_{i}\right)-2 .
$$

Here, $a_{i}$ 's are integers satisfying $8 \mid a_{i}$ and some other conditions. (These types of polynomials are also dealt with in [5].) We state the main result (Proposition 3) in Section 2, and show it in Section 3. Applying Proposition 3 and Theorem 1, we examine whether $F(\mathfrak{l}) / F$ has an NIB in (Proposition 6 of) Section 4. The final section is of supplementary nature. First, we show that the above mentioned family of totally real number fields of degree $n$ contains infinite ones (Proposition 7). Next, we give an assertion (Proposition 9) on Galois extensions of prime power degree. As its consequence, we see that when $n=3$, the cubic fields in this article are not cyclic ones which are dealt with in [7].

Acknowledgments. The second author expresses his appreciation to Yoshitaka Odai for suggesting Proposition 9.

2. Main result. We introduce a family of totally real number fields of Eisenstein type. Let $n \geq 2$ be a positive integer and take $n-1$ odd primes $p_{i}(2 \leq i \leq n)$ such that

$$
p_{i} \equiv 5 \bmod 8, \quad p_{i} \nmid(2 n-1) .
$$

Furthermore, let $a_{1}, \ldots, a_{n}$ be integers which satisfy the conditions (2.2)-

$$
1 \leq i<j \leq n \Rightarrow a_{j}-a_{i}>2 \sqrt[n]{2},
$$

and for each $i(1 \leq i \leq n)$,

$$
\begin{aligned}
& a_{i} \equiv 0 \bmod 8, \\
& a_{i} \equiv-1 \bmod p_{i} \quad \text { if } i \neq 1, \\
& a_{i} \equiv 0 \bmod p_{j} \quad \text { for all } j(2 \leq j \leq n, j \neq i) .
\end{aligned}
$$


Then we put

$$
f(X):=\prod_{i=1}^{n}\left(X-a_{i}\right)-2 .
$$

Since $f(X) \equiv X^{n}-2 \bmod 4$ by $(2.3), f(X)$ is an Eisenstein polynomial for 2 . Let $\theta$ be a root of $f(X)$, and define

$$
F:=\mathbb{Q}(\theta),
$$

where $\mathbb{Q}$ denotes the field of all rational numbers. As we shall show at the end of this section, (2.2) and the intermediate value theorem imply that $f(X)$ has $n$ distinct real roots $\theta_{i}(1 \leq i \leq n)$ satisfying the following: when $n$ is even,

$$
\theta_{1}<a_{1}<a_{2}<\theta_{2}<\theta_{3}<\ldots<\theta_{n-2}<\theta_{n-1}<a_{n-1}<a_{n}<\theta_{n}
$$

when $n$ is odd,

$$
a_{1}<\theta_{1}<\theta_{2}<a_{2}<a_{3}<\ldots<\theta_{n-2}<\theta_{n-1}<a_{n-1}<a_{n}<\theta_{n} .
$$

In particular, $F$ is totally real. Also, 2 is totally ramified in $F: 2 \mathfrak{o}_{F}=\mathfrak{p}^{n}$. As $a_{i}(1 \leq i \leq n)$ is even, we have $\operatorname{ord}_{\mathfrak{p}}(\theta)=1<\operatorname{ord}_{\mathfrak{p}}\left(a_{i}\right)$, so that $\operatorname{ord}_{\mathfrak{p}}\left(\theta-a_{i}\right)$ $=1$; also, we have $\prod_{i=1}^{n}\left(\theta-a_{i}\right)=2$ since $f(\theta)=0$. Hence, $\mathfrak{p}=\left(\theta-a_{i}\right) \mathfrak{o}_{F}$ for all $i(1 \leq i \leq n)$. Therefore,

$$
\varepsilon_{i}:=\frac{\theta-a_{i}}{\theta-a_{1}}
$$

$(2 \leq i \leq n)$ are elements of $\mathfrak{o}_{F}^{\times}$, and (2.3) implies that $\varepsilon_{i} \equiv 1 \bmod 4$. By (2.1), (2.4) and (2.5), Lemma 2 follows from the same argument as in the proof of $[5$, Lemma].

LEMMA 2. Under the above setting, $\left\{[-1],\left[\varepsilon_{i}\right] \mid 2 \leq i \leq n\right\}$ is an $\mathbb{F}_{2}$-basis of $\mathfrak{o}_{F}^{\times} / \mathfrak{o}_{F}^{\times 2}$.

For each $i(1 \leq i \leq n)$, we define a real embedding $\iota_{i}$ of $F$ by putting $\iota_{i}(\theta):=\theta_{i}$. Let $\mathfrak{l}_{i}$ be the real prime of $F$ corresponding to $\iota_{i}$. We have $\mathfrak{l}_{0}=\mathfrak{l}_{1} \mathfrak{l}_{2} \ldots \mathfrak{l}_{n}$. For $\mathfrak{l} \mid \mathfrak{l}_{0}$, we define a group $\bar{E}^{\mathfrak{l}}$ by

$$
\bar{E}^{\mathfrak{l}}:=\left\{[\eta] \in \mathfrak{o}_{F}^{\times} / \mathfrak{o}_{F}^{\times 2} \mid \eta \in \mathfrak{o}_{F}^{\times}, \iota_{i}(\eta)>0 \text { for all } \mathfrak{l}_{i} \mid \mathfrak{l}_{0} \mathfrak{l}^{-1}\right\},
$$

which we also regard as a vector space over $\mathbb{F}_{2}$. The vector space $\mathcal{N}^{r}$ is a subspace of $\bar{E}^{\mathfrak{l}}$. We determine $\mathbb{F}_{2}$-bases of $\mathcal{N}^{\mathfrak{l}}$ and of $\bar{E}^{\mathfrak{l}}$, respectively, in Proposition 3 which we show in the next section.

Definition 2.1. Let $\mathfrak{l} \mid \mathfrak{l}_{0}$. When $n$ is even (resp. odd), we define

$$
\begin{aligned}
S=S^{\mathfrak{l}}:=\{k \mid 1 & \leq k \leq(n-2) / 2(\operatorname{resp} .(n-1) / 2), \\
i & \left.=2 k \text { or } 2 k+1(\text { resp. } 2 k-1 \text { or } 2 k) \text { with some } \mathfrak{l}_{i} \mid \mathfrak{l}_{0} \mathfrak{l}^{-1}\right\}
\end{aligned}
$$

and put $\sigma=\sigma^{\mathfrak{l}}:=|S|$. We write $S=\left\{k_{1}, \ldots, k_{\sigma}\right\}$ with $k_{1}<\ldots<k_{\sigma}$. 
Proposition 3. Let $F$ be a totally real number field as in (2.6) of degree $n$ and $\varepsilon_{i}(2 \leq i \leq n)$ units of $F$ as in (2.9). Let $\mathfrak{l} \mid \mathfrak{l}_{0}$. Then, under the notation of Definition 2.1, we have $\operatorname{dim} \mathcal{N}^{\mathfrak{l}}=n-1-\sigma^{\mathfrak{l}}$. Furthermore, the following hold (when $n=2$, we put $k_{0}:=0$ and $\varepsilon_{1}:=1$ ):

(i) Suppose that $n$ is even, and put

$$
\begin{aligned}
& A_{0}:=\left\{\left[\varepsilon_{i}\right] \mid 2 \leq i \leq 2 k_{1}\right\}, \\
& B_{0}:=\left\{\left[\varepsilon_{i} \varepsilon_{2 k_{s+1}}\right] \mid 1 \leq s \leq \sigma-1,2 k_{s}+1 \leq i \leq 2 k_{s+1}-1\right\}, \\
& C_{0}:=\left\{\left[-\varepsilon_{i}\right] \mid 2 k_{\sigma}+1 \leq i \leq n\right\}, \\
& D_{0}:=\left\{\left[\varepsilon_{i} \varepsilon_{n}\right] \mid 2 k_{\sigma}+1 \leq i \leq n-1\right\} .
\end{aligned}
$$

If $\mathfrak{l}_{1} \mathfrak{l}_{n} \mid \mathfrak{l}$, then $A_{0} \cup B_{0} \cup C_{0}$ (resp. $\left.A_{0} \cup B_{0} \cup D_{0}\right)$ is an $\mathbb{F}_{2}$-basis of $\bar{E}^{\mathfrak{l}}$ $\left(\right.$ resp. $\left.\mathcal{N}^{\mathfrak{l}}\right)$. In particular, $\operatorname{dim} \bar{E}^{\mathfrak{l}}=n-\sigma^{\mathfrak{l}}$. If $\mathfrak{l}_{1} \mathfrak{l}_{n} \nmid \mathfrak{l}$, then $\bar{E}^{\mathfrak{l}}=\mathcal{N}^{\mathfrak{l}}$, and $A_{0} \cup B_{0} \cup D_{0}$ is an $\mathbb{F}_{2}$-basis of $\mathcal{N}^{\mathfrak{r}}$.

(ii) Suppose that $n$ is odd, and put

$$
\begin{aligned}
& A_{1}:=\left\{\left[\varepsilon_{i}\right] \mid 2 \leq i \leq 2 k_{1}-1\right\}, \\
& B_{1}:=\left\{\left[\varepsilon_{i} \varepsilon_{2 k_{s+1}-1}\right] \mid 1 \leq s \leq \sigma-1,2 k_{s} \leq i \leq 2 k_{s+1}-2\right\}, \\
& C_{1}:=\left\{\left[-\varepsilon_{i}\right] \mid 2 k_{\sigma} \leq i \leq n\right\}, \\
& D_{1}:=\left\{\left[\varepsilon_{i} \varepsilon_{n}\right] \mid 2 k_{\sigma} \leq i \leq n-1\right\} .
\end{aligned}
$$

If $\mathfrak{l}_{n} \mid \mathfrak{l}$, then $A_{1} \cup B_{1} \cup C_{1}$ (resp. $\left.A_{1} \cup B_{1} \cup D_{1}\right)$ is an $\mathbb{F}_{2}$-basis of $\bar{E}^{\mathfrak{l}}\left(\right.$ resp. $\left.\mathcal{N}^{\mathfrak{l}}\right)$. In particular, $\operatorname{dim} \bar{E}^{\mathfrak{l}}=n-\sigma^{\mathfrak{l}}$. If $\mathfrak{l}_{n} \nmid \mathfrak{l}$, then $\bar{E}^{\mathfrak{l}}=\mathcal{N}^{\mathfrak{l}}$, and $A_{1} \cup B_{1} \cup D_{1}$ is an $\mathbb{F}_{2}$-basis of $\mathcal{N}^{\mathfrak{l}}$.

Since $\varepsilon_{i} \equiv 1 \bmod 4$ for all $i$, Theorem $1(\mathrm{i})$ and Proposition 3 yield:

Corollary 4. Let the assumption and notation be as in Proposition 3. Then an element $x$ of the following form is a generator of NIB of $L^{\mathfrak{l}} / F$ : when $n$ is even,

$$
x=\prod_{i=2}^{2 k_{1}}\left(\frac{1+\sqrt{\varepsilon_{i}}}{2}\right) \prod_{s=1}^{\sigma-1} \prod_{i=2 k_{s}+1}^{2 k_{s+1}-1}\left(\frac{1+\sqrt{\varepsilon_{i} \varepsilon_{2 k_{s+1}}}}{2}\right) \prod_{i=2 k_{\sigma}+1}^{n-1}\left(\frac{1+\sqrt{\varepsilon_{i} \varepsilon_{n}}}{2}\right) ;
$$

when $n$ is odd,

$$
x=\prod_{i=2}^{2 k_{1}-1}\left(\frac{1+\sqrt{\varepsilon_{i}}}{2}\right) \prod_{s=1}^{\sigma-1} \prod_{i=2 k_{s}}^{2 k_{s+1}-2}\left(\frac{1+\sqrt{\varepsilon_{i} \varepsilon_{2 k_{s+1}-1}}}{2}\right) \prod_{i=2 k_{\sigma}}^{n-1}\left(\frac{1+\sqrt{\varepsilon_{i} \varepsilon_{n}}}{2}\right) .
$$

EXAMPLE 2.2. When $n$ is even and $1 \leq k \leq(n-2) / 2$, we $\operatorname{list} \operatorname{dim} \mathcal{N}^{\mathfrak{l}}$ for some $\mathfrak{l}$ in Table I. 
Table I

\begin{tabular}{lcc}
\hline $\mathfrak{l}$ & $\sigma^{\mathfrak{l}}$ & $\operatorname{dim} \mathcal{N}^{\mathfrak{l}}$ \\
\hline $\mathfrak{l}_{0}$ & 0 & $n-1$ \\
$\mathfrak{l}_{0} \mathfrak{l}_{1}^{-1} \mathfrak{l}_{n}^{-1}$ & 0 & $n-1$ \\
$\mathfrak{l}_{2} \mathfrak{l}_{3} \mathfrak{l}_{4} \ldots \mathfrak{l}_{2 k+1}$ & $(n-2) / 2-k$ & $n / 2+k$ \\
$\mathfrak{l}_{2} \mathfrak{l}_{4} \mathfrak{l}_{6} \ldots \mathfrak{l}_{2 k}$ & $(n-2) / 2$ & $n / 2$ \\
$\mathfrak{l}_{1} \mathfrak{l}_{n}$ & $(n-2) / 2$ & $n / 2$ \\
1 & $(n-2) / 2$ & $n / 2$ \\
\hline
\end{tabular}

ExAmple 2.3. When $n(\geq 3)$ is odd and $1 \leq k \leq(n-1) / 2$, we list $\operatorname{dim} \mathcal{N}^{\mathfrak{l}}$ for some $\mathfrak{l}$ in Table II.

Table II

\begin{tabular}{lcc}
\hline $\mathfrak{l}$ & $\sigma^{\mathfrak{l}}$ & $\operatorname{dim} \mathcal{N}^{\mathfrak{l}}$ \\
\hline $\mathfrak{l}_{0}$ & 0 & $n-1$ \\
$\mathfrak{l}_{0} \mathfrak{l}_{n}^{-1}$ & 0 & $n-1$ \\
$\mathfrak{l}_{1} \mathfrak{l}_{2} \mathfrak{l}_{3} \ldots \mathfrak{l}_{2 k}$ & $(n-1) / 2-k$ & $(n-1) / 2+k$ \\
$\mathfrak{l}_{1} \mathfrak{l}_{3} \mathfrak{l}_{5} \ldots \mathfrak{l}_{2 k-1}$ & $(n-1) / 2$ & $(n-1) / 2$ \\
$\mathfrak{l}_{n}$ & $(n-1) / 2$ & $(n-1) / 2$ \\
1 & $(n-1) / 2$ & $(n-1) / 2$ \\
\hline
\end{tabular}

In order to prove (2.8), we assume that $n$ is odd, and let $1 \leq i \leq n$. Then $f\left(a_{i}\right)=-2<0$. If $i$ is odd, since $n-i$ is even, (2.2) implies that

$$
\begin{aligned}
f\left(a_{i}+\sqrt[n]{2}\right) & =\prod_{j=1}^{i}\left(a_{i}-a_{j}+\sqrt[n]{2}\right) \prod_{j=i+1}^{n}\left(a_{j}-a_{i}-\sqrt[n]{2}\right)-2 \\
& >\sqrt[n]{2}(3 \sqrt[n]{2})^{i-1} \cdot(\sqrt[n]{2})^{n-i}-2 \geq(\sqrt[n]{2})^{n}-2=0 .
\end{aligned}
$$

Hence, the intermediate value theorem shows that $f(X)$ has a real root in the open interval $\left(a_{i}, a_{i}+\sqrt[n]{2}\right)$. If $i$ is even, since $n-(i-1)$ is even, the same argument implies that $f\left(a_{i}-\sqrt[n]{2}\right)>0$. Consequently, $f(X)$ has a real root in $\left(a_{i}-\sqrt[n]{2}, a_{i}\right)$. This implies the condition (2.8), and similarly we obtain (2.7).

3. Proof of Proposition 3. In this section we prove Proposition 3. Let $2 \leq i \leq n$. Then we claim the following: when $n$ is even and $1 \leq k \leq$ $(n-2) / 2$,

$$
\begin{gathered}
\iota_{1}\left(\varepsilon_{i}\right)>0, \iota_{n}\left(\varepsilon_{i}\right)>0 ; \quad \iota_{2 k}\left(\varepsilon_{i}\right)>0, \iota_{2 k+1}\left(\varepsilon_{i}\right)>0 \quad \text { if } i \leq 2 k ; \\
\iota_{2 k}\left(\varepsilon_{i}\right)<0, \iota_{2 k+1}\left(\varepsilon_{i}\right)<0 \quad \text { if } i \geq 2 k+1 ;
\end{gathered}
$$


when $n$ is odd and $1 \leq k \leq(n-1) / 2$,

$$
\begin{gathered}
\iota_{n}\left(\varepsilon_{i}\right)>0 ; \quad \iota_{2 k-1}\left(\varepsilon_{i}\right)>0, \iota_{2 k}\left(\varepsilon_{i}\right)>0 \quad \text { if } i \leq 2 k-1 ; \\
\iota_{2 k-1}\left(\varepsilon_{i}\right)<0, \iota_{2 k}\left(\varepsilon_{i}\right)<0 \quad \text { if } i \geq 2 k .
\end{gathered}
$$

These are shown as follows. For each $j(1 \leq j \leq n)$, we have $\iota_{j}\left(\varepsilon_{i}\right)=$ $\left(\theta_{j}-a_{i}\right) /\left(\theta_{j}-a_{1}\right)$. Assume that $n$ is even and $1 \leq k \leq(n-2) / 2$. Since $\theta_{1}<a_{1}<a_{2} \leq a_{i}$ by (2.7), we obtain $\iota_{1}\left(\varepsilon_{i}\right)>0$; also, $a_{1}<a_{i}<\theta_{n}$ implies that $\iota_{n}\left(\varepsilon_{i}\right)>0$. Furthermore, we have

$$
a_{2 k}<\theta_{2 k}<\theta_{2 k+1}<a_{2 k+1}<a_{2 k+2}
$$

from (2.7). Hence, if $i \leq 2 k$ (resp., $i>2 k$ ), as $a_{1}<\theta_{2 k}$, we have $\iota_{2 k}\left(\varepsilon_{i}\right)>0$ and $\iota_{2 k+1}\left(\varepsilon_{i}\right)>0$ (resp., $\iota_{2 k}\left(\varepsilon_{i}\right)<0$ and $\iota_{2 k+1}\left(\varepsilon_{i}\right)<0$ ). Thus (3.1) holds. Similarly, (3.2) follows from (2.8).

Lemma 2 shows that

$$
\mathcal{N}^{\mathfrak{l}_{0}}=\prod_{i=2}^{n}\left\langle\left[\varepsilon_{i}\right]\right\rangle \quad \text { and } \quad \mathfrak{o}_{F}^{\times} / \mathfrak{o}_{F}^{\times 2}=\langle[-1]\rangle \times \mathcal{N}^{\mathfrak{l}_{0}},
$$

because $\varepsilon_{i} \equiv 1 \bmod 4(2 \leq i \leq n)$ and $-1 \not \equiv 1 \bmod 4$. In the remainder of the proof, we let $[\eta] \in \mathfrak{o}_{F}^{\times} / \mathfrak{o}_{F}^{\times 2}$, and write $[\eta]=[-1]^{e_{1}} \prod_{i=2}^{n}\left[\varepsilon_{i}\right]^{e_{i}}$ with some $e_{1}, e_{i}$ in $\{0,1\}$. It follows immediately from (3.3) that

$$
[\eta] \in \mathcal{N}^{\mathfrak{l}}\left(\subset \mathcal{N}^{\mathfrak{l}_{0}}\right) \Rightarrow e_{1}=0 .
$$

If $1 \leq j \leq n$, then $\left[\iota_{j}(\eta)\right]=[-1]^{e_{1}} \prod_{i=2}^{n}\left[\iota_{j}\left(\varepsilon_{i}\right)\right]^{e_{i}}$. We prove only the assertion (i) of Proposition 3, since the same argument implies (ii). By (3.1), if $j=2 k$ or $2 k+1$, then

$$
\iota_{j}(\eta)>0 \Leftrightarrow e_{1}+\sum_{i=2 k+1}^{n} e_{i} \equiv 0 \bmod 2 .
$$

It follows from this and the definition of $S^{\mathfrak{l}}$ that

$$
\begin{aligned}
{[\eta] \in \bar{E}^{\mathrm{l}} \Leftrightarrow } & e_{1}+\sum_{i=2 k_{s}+1}^{n} e_{i} \equiv 0 \bmod 2 \text { for all } s(1 \leq s \leq \sigma) \\
\Leftrightarrow & \sum_{i=2 k_{s}+1}^{2 k_{s+1}} e_{i} \equiv 0 \bmod 2 \text { for all } s(1 \leq s \leq \sigma-1), \\
& \text { and } e_{1}+\sum_{i=2 k_{\sigma}+1}^{n} e_{i} \equiv 0 \bmod 2 .
\end{aligned}
$$

First, assume that $\mathfrak{l}_{1} \mathfrak{l}_{n} \mid \mathfrak{l}$, and $[\eta] \in \bar{E}^{\mathfrak{l}}$ (resp., $\in \mathcal{N}^{\mathfrak{l}}$ ). Then (3.5) and (3.4) imply that $e_{2 k_{s+1}} \equiv \sum_{i=2 k_{s}+1}^{2 k_{s+1}-1} e_{i}$ for all $s(1 \leq s \leq \sigma-1)$, and $e_{1} \equiv$ 
$\sum_{i=2 k_{\sigma}+1}^{n} e_{i} \bmod 2$ (resp., $\left.e_{n} \equiv \sum_{i=2 k_{\sigma}+1}^{n-1} e_{i} \bmod 2\right)$. Hence,

$$
\begin{aligned}
{[\eta]=\prod_{i=2}^{2 k_{1}}\left[\varepsilon_{i}\right]^{e_{i}} \prod_{s=1}^{\sigma-1} \prod_{i=2 k_{s}+1}^{2 k_{s+1}-1}\left[\varepsilon_{i} \varepsilon_{2 k_{s+1}}\right]^{e_{i}} \times \prod_{i=2 k_{\sigma}+1}^{n}\left[-\varepsilon_{i}\right]^{e_{i}} } \\
\left(\text { resp. } \times \prod_{i=2 k_{\sigma}+1}^{n-1}\left[\varepsilon_{i} \varepsilon_{n}\right]^{e_{i}}\right) .
\end{aligned}
$$

Also, all elements of $A_{0} \cup B_{0} \cup C_{0}$ (resp. $\left.A_{0} \cup B_{0} \cup D_{0}\right)$ are in $\bar{E}^{\mathfrak{l}}$ (resp. $\mathcal{N}^{\mathfrak{l}}$ ) by (3.5), and are linearly independent over $\mathbb{F}_{2}$ by (3.3). Therefore this set constitutes an $\mathbb{F}_{2}$-basis of $\bar{E}^{\mathfrak{l}}\left(\operatorname{resp} . \mathcal{N}^{\mathfrak{l}}\right)$. So,

$$
\operatorname{dim} \bar{E}^{\mathfrak{l}}=\left(2 k_{1}-1\right)+\sum_{s=1}^{\sigma-1}\left(2 k_{s+1}-2 k_{s}-1\right)+\left(n-2 k_{\sigma}\right)=n-\sigma .
$$

Similarly, we have $\operatorname{dim} \mathcal{N}^{\mathfrak{l}}=n-1-\sigma$.

Next, assume that $\mathfrak{l}_{1} \mathfrak{l}_{n} \nmid \mathfrak{l}$ and $[\eta] \in \bar{E}^{\mathfrak{l}}$. Then $\iota_{1}(\eta)>0$ or $\iota_{n}(\eta)>0$; therefore we have $e_{1}=0$ by (3.1). Hence, (3.3) implies that $\bar{E}^{\mathfrak{l}}=\mathcal{N}^{\mathfrak{l}}$. By the same argument as above, $A_{0} \cup B_{0} \cup D_{0}$ is an $\mathbb{F}_{2}$-basis of $\mathcal{N}^{\mathfrak{l}}$. This proves (i).

4. NIB of $F(\mathfrak{l}) / F$. In this section, using Proposition 3, we examine whether $F(\mathfrak{l}) / F$ has an NIB. We assume that $F$ is a totally real number field as in (2.6) of degree $n$, and $\mathfrak{l}_{i}(1 \leq i \leq n)$ is the real prime of $F$ corresponding to the real embedding $\iota_{i}$, defined in Section 2. For $\mathfrak{l} \mid \mathfrak{l}_{0}$, let $\varrho_{\mathfrak{l}}$ denote the number of distinct prime divisors of $\mathfrak{l}$. Then the Galois group $\operatorname{Gal}(F(\mathfrak{l}) / F(1))$ is an elementary abelian 2-group, which is also regarded as a vector space over $\mathbb{F}_{2}$. We have

$$
\delta_{\mathfrak{l}}:=\operatorname{dim} \operatorname{Gal}(F(\mathfrak{l}) / F(1))=\operatorname{dim} \bar{E}^{\mathfrak{l}_{0} \mathfrak{l}^{-1}}-\varrho_{\mathfrak{l}_{0} \mathfrak{l}-1}
$$

(cf. [7, Section 3]).

LEMMA 5. Let $\mathfrak{l} \mid \mathfrak{l}_{0}$.

(i) When $n$ is even, if $\mathfrak{l} \mid \mathfrak{l}_{0} \mathfrak{l}_{1}^{-1} \mathfrak{l}_{n}^{-1}$ then $\delta_{\mathfrak{l}}=\varrho_{\mathfrak{l}}-\sigma^{\mathfrak{l}_{0} \mathfrak{l}^{-1}}$, and otherwise $\delta_{\mathfrak{l}}=\varrho_{\mathfrak{l}}-\sigma^{\mathfrak{l}_{0} \mathfrak{l}^{-1}}-1$.

(ii) When $n$ is odd, if $\mathfrak{l} \mid \mathfrak{l}_{0} \mathfrak{l}_{n}^{-1}$ then $\delta_{\mathfrak{l}}=\varrho_{\mathfrak{l}}-\sigma^{\mathfrak{l}_{0} \mathfrak{l}^{-1}}$, and otherwise $\delta_{\mathfrak{l}}=$ $\varrho \mathfrak{l}-\sigma^{\mathfrak{l}_{0} \mathfrak{l}^{-1}}-1$.

Proof. Using Proposition 3, we can calculate $\delta_{\mathfrak{l}}$ from (4.1). If $\mathfrak{l} \mid \mathfrak{l}_{0} \mathfrak{l}_{1}^{-1} \mathfrak{l}_{n}^{-1}$, since $\mathfrak{l}_{1} \mathfrak{l}_{n} \mid \mathfrak{l}_{0} \mathfrak{l}^{-1}$, we have $\delta_{\mathfrak{l}}=\left(n-\sigma^{\mathfrak{l}_{0} \mathfrak{l}^{-1}}\right)-\varrho_{\mathfrak{l}_{0} \mathfrak{l}^{-1}}=\varrho_{\mathfrak{l}}-\sigma^{\mathfrak{l}_{0} \mathfrak{l}^{-1}}$. If $\mathfrak{l} \nmid \mathfrak{l}_{0} \mathfrak{l}_{1}^{-1} \mathfrak{l}_{n}^{-1}$, then $\delta_{\mathfrak{l}}=\left(n-1-\sigma^{\mathfrak{l}_{0} \mathfrak{l}^{-1}}\right)-\varrho_{\mathfrak{l}_{0} \mathfrak{l}^{-1}}=\varrho_{\mathfrak{l}}-\sigma^{\mathfrak{l}_{0} \mathfrak{l}^{-1}}-1$. Hence we obtain $(\mathrm{i})$; the proof of (ii) is similar.

Proposition 6. We have 2-rank $\operatorname{Gal}(F(1) / F) \geq[n / 2]$, where $[\alpha]$ denotes the largest integer not exceeding a real number $\alpha$. Furthermore, let 
$\mathfrak{l} \mid \mathfrak{l}_{0}$. Then:

(i) When " $n$ is even and $\mathfrak{l}_{1} \mathfrak{l}_{n} \nmid \mathfrak{l}$ ", or $n$ is odd, we have: $F(\mathfrak{l}) / F$ has an NIB if and only if $h_{F}=2^{[n / 2]}$.

(ii) When $n$ is even and $\mathfrak{l}_{1} \mathfrak{l}_{n} \mid \mathfrak{l}, F(\mathfrak{l}) / F$ has no $N I B$.

Proof. Since $L^{1}$ is a subfield of $F(1)$, we have

$$
\text { 2-rank } \operatorname{Gal}(F(1) / F) \geq 2 \text {-rank } \operatorname{Gal}\left(L^{1} / F\right)=\operatorname{dim} \mathcal{N}^{1} .
$$

Examples 2.2 and 2.3 imply that $\operatorname{dim} \mathcal{N}^{1}=[n / 2]$. Therefore we obtain 2 -rank $\operatorname{Gal}(F(1) / F) \geq[n / 2]$. If we write $\left|\mathcal{N}^{\mathfrak{l}}\right|[F(\mathfrak{l}): F(1)]^{-1}=2^{e_{\mathfrak{r}}}$ with some integer $e_{\mathfrak{l}}$, then Proposition 3 yields

$$
e_{\mathfrak{l}}=n-1-\left(\sigma^{\mathfrak{l}}+\delta_{\mathfrak{l}}\right) \text {. }
$$

As before, let $S=S^{\mathfrak{l}}$ and $\sigma=\sigma^{\mathfrak{l}}$. For brevity, put $S^{\prime}:=S^{\mathfrak{l}_{0} \mathfrak{l}^{-1}}, \sigma^{\prime}:=\left|S^{\prime}\right|$, and $t:=\left|S \cap S^{\prime}\right|$. To show Proposition 6, we first write $\varrho_{\mathfrak{l}}$ and $\varrho_{\mathfrak{l}_{0} \mathfrak{l}^{-1}}$ in terms of $\sigma, \sigma^{\prime}, t$ (and next calculate $e_{\mathfrak{l}}$ ). For this, the following remark is useful. When $n$ is even, we see from the definition of $S$ and $S^{\prime}$ that (I) if $k \in S \cap S^{\prime}$ then either "ll $\mathfrak{l}_{2 k} \mid \mathfrak{l}_{0} \mathfrak{l}^{-1}$ and $\mathfrak{l}_{2 k+1} \mid \mathfrak{l}$ ", or " $\mathfrak{l}_{2 k+1} \mid \mathfrak{l}_{0} \mathfrak{l}^{-1}$ and $\mathfrak{l}_{2 k} \mid \mathfrak{l}$ ", and that (II) if $k \in S-\left(S \cap S^{\prime}\right)$ (resp., $\in S^{\prime}-\left(S \cap S^{\prime}\right)$ ), then $\mathfrak{l}_{2 k} \mathfrak{l}_{2 k+1} \mid \mathfrak{l}_{0} \mathfrak{l}^{-1}$ (resp., $\left.\mathfrak{l}_{2 k} \mathfrak{l}_{2 k+1} \mid \mathfrak{l}\right)$. A similar assertion holds for $n$ odd.

(A) The case where $n$ is even. When $\mathfrak{l} \nmid \mathfrak{l}_{0} \mathfrak{l}_{1}^{-1} \mathfrak{l}_{n}^{-1}$, we put $u:=\mid\{1, n\} \cap$ $\left\{i ; \mathfrak{l}_{i} \mid \mathfrak{l}\right\} \mid$. If $\mathfrak{l} \mid \mathfrak{l}_{0} \mathfrak{l}_{1}^{-1} \mathfrak{l}_{n}^{-1}$ (resp., $\mathfrak{l} \nmid \mathfrak{l}_{0} \mathfrak{l}_{1}^{-1} \mathfrak{l}_{n}^{-1}$ ), by the above remark, we have $\varrho_{\mathfrak{l}_{0} \mathfrak{l}-1}=t+2(\sigma-t)+2$ and $\varrho_{\mathfrak{l}}=t+2\left(\sigma^{\prime}-t\right)\left(\operatorname{resp} ., \varrho_{\mathfrak{l}_{0} \mathfrak{l}-1}=t+2(\sigma-t)+2-u\right.$ and $\left.\varrho_{\mathfrak{l}}=t+2\left(\sigma^{\prime}-t\right)+u\right)$. Consequently, $\varrho_{\mathfrak{l}_{0} \mathfrak{l}-1}-\varrho_{\mathfrak{l}}=2\left(\sigma-\sigma^{\prime}\right)+2$ (resp., $\left.\varrho_{\mathfrak{l} \mathfrak{l}^{-1}}-\varrho_{\mathfrak{l}}=2\left(\sigma-\sigma^{\prime}\right)+2-2 u\right)$. On the other hand, we clearly have $\varrho_{\mathfrak{l}_{0} \mathfrak{l}^{-1}}+\varrho_{\mathfrak{l}}=n$. Therefore, we obtain

$$
(n-2) / 2(\text { resp. } n / 2+u-1)=\varrho_{\mathfrak{l}}+\sigma-\sigma^{\prime} .
$$

By (4.2) and Lemma 5(i), we obtain $e_{\mathfrak{r}}=n-1-\left(\sigma+\varrho_{\mathfrak{l}}-\sigma^{\prime}\right)$ (resp., $\left.=n-\left(\sigma+\varrho_{\mathfrak{l}}-\sigma^{\prime}\right)\right)$. Hence, (4.3) implies that $e_{\mathfrak{l}}=n / 2(\operatorname{resp} .,=n / 2-(u-1))$. Since $u=1$ or 2 , if $\mathfrak{l}_{1} \mathfrak{l}_{n} \nmid \mathfrak{l}$ (resp., $\left.\mathfrak{l}_{1} \mathfrak{l}_{n} \mid \mathfrak{l}\right)$ then $e_{\mathfrak{l}}=n / 2$ (resp., $=n / 2-1$ ).

(B) The case where $n$ is odd. If $\mathfrak{l} \mid \mathfrak{l}_{0} \mathfrak{l}_{n}^{-1}$ (resp., $\mathfrak{l} \nmid \mathfrak{l}_{0} \mathfrak{l}_{n}^{-1}$ ), by the above remark, we have $\varrho_{\mathfrak{l}_{0} \mathfrak{l}^{-1}}=t+2(\sigma-t)+1$ and $\varrho_{\mathfrak{l}}=t+2\left(\sigma^{\prime}-t\right)$ (resp., $\varrho_{\mathfrak{l}_{0} \mathfrak{l}^{-1}}=t+2(\sigma-t)$ and $\left.\varrho_{\mathfrak{l}}=t+2\left(\sigma^{\prime}-t\right)+1\right)$; consequently, $\varrho_{\mathfrak{l}_{0}} \mathfrak{l}^{-1}-\varrho_{\mathfrak{l}}=$ $2\left(\sigma-\sigma^{\prime}\right)+1$ (resp., $\left.\varrho_{\mathfrak{l}_{0} \mathfrak{l}^{-1}}-\varrho_{\mathfrak{l}}=2\left(\sigma-\sigma^{\prime}\right)-1\right)$; therefore,

$$
(n-1) / 2(\operatorname{resp} .(n+1) / 2)=\varrho_{\mathfrak{l}}+\sigma-\sigma^{\prime} .
$$

By (4.2) and Lemma 5(ii), we obtain $e_{\mathfrak{l}}=n-1-\left(\sigma+\varrho_{\mathfrak{l}}-\sigma^{\prime}\right)$ (resp., $\left.=n-\left(\sigma+\varrho_{\mathfrak{l}}-\sigma^{\prime}\right)\right)$. Hence, (4.4) implies that $e_{\mathfrak{l}}=(n-1) / 2$.

Theorem 1(ii) shows that $F(\mathfrak{l}) / F$ has an NIB if and only if $h_{F}=2^{e_{\mathfrak{l}}}$. When " $n$ is even and $\mathfrak{l}_{1} \mathfrak{l}_{n} \nmid \mathfrak{l}$ ", or $n$ is odd, $(\mathrm{A})$ and $(\mathrm{B})$ imply that $e_{\mathfrak{l}}=[n / 2]$. Hence the assertion (i) holds. When $n$ is even and $\mathfrak{l}_{1} \mathfrak{l}_{n} \mid \mathfrak{l}$, since $2^{[n / 2]} \mid h_{F}$, it 
follows from (A) that

$$
2^{e_{\mathrm{r}}}=2^{n / 2-1}<2^{[n / 2]} \leq h_{F} .
$$

Hence $F(\mathfrak{l}) / F$ has no NIB. This proves our proposition.

Assume that $h_{F}=2^{[n / 2]}$. When $n$ is even, Proposition 6 implies that $F(\mathfrak{l}) / F$ has an NIB if and only if $\mathfrak{l}_{1} \mathfrak{l}_{n} \nmid \mathfrak{l}$. Also, when $n$ is odd, $F(\mathfrak{l}) / F$ has an NIB for all $\mathfrak{l} \mid \mathfrak{l}_{0}$. On the other hand, if $h_{F} \neq 2^{[n / 2]}$ then $F(\mathfrak{l}) / F$ has no NIB for all $\mathfrak{l} \mid \mathfrak{l}_{0}$. Thus the existence of NIB of $F(\mathfrak{l}) / F$ is determined by the condition on the class number $h_{F}$ and an integral divisor $\mathfrak{l}$.

Remark 4.1. Suppose that $n=2$. Let $\varepsilon(>1)$ be the fundamental unit of $F$ and $g$ the order of $\varepsilon \bmod 4$ in $\left(\mathfrak{o}_{F} / 4 \mathfrak{o}_{F}\right)^{\times}$. By Lemma 2, we see that the index $\left(\mathfrak{o}_{F}^{\times}:\langle-1\rangle \times\left\langle\varepsilon_{2}\right\rangle\right)$ is odd, where the unit $\varepsilon_{2}$ is defined in (2.9). This implies that $g$ is odd and $\varepsilon$ is totally positive. Hence, Proposition 6 for $n=2$ also follows from [7, Corollaries 11 and 12].

EXAMPLE 4.2. Let $C l_{F}$ be the ideal class group of $F$. For a positive integer $m$, we denote by $C_{m}$ a cyclic group of order $m$. We consider a real quadratic field $F$ which is defined by a polynomial of the form $f(X)=$ $X\left(X-a_{2}\right)-2$, where $a_{2}$ is an integer such that $a_{2} \equiv 0 \bmod 8$ and $a_{2} \equiv$ $-1 \bmod p_{2}, p_{2}$ being a prime with $p_{2} \equiv 5 \bmod 8$. For all fields $F$ in Table III, by using PARI [1], we see that $F(1) / F$ has a relative integral basis, that is, $\mathfrak{o}_{F(1)}$ has a free $\mathfrak{o}_{F}$-basis; we can also obtain the same result by using KASH [8] (cf. [7, Section 5]). But, as $h_{F} \neq 2, F(1) / F$ has no NIB by Proposition 6.

Table III

\begin{tabular}{rccr}
\hline$p_{2}$ & $a_{2}$ & $C l_{F}$ & $h_{F}$ \\
\hline 5 & 224 & $C_{2} \times C_{2}$ & 4 \\
5 & 424 & $C_{6}$ & 6 \\
5 & 54744 & $C_{2} \times C_{2}$ & 4 \\
5 & 138944 & $C_{2} \times C_{2}$ & 4 \\
5 & 156624 & $C_{2} \times C_{2}$ & 4 \\
13 & 168 & $C_{6}$ & 6 \\
13 & 13896 & $C_{6}$ & 6 \\
29 & 11512 & $C_{2} \times C_{2}$ & 4 \\
157 & 23392 & $C_{2} \times C_{2}$ & 4 \\
\hline
\end{tabular}

5. Supplements. In this section we prove Propositions 7 and 9.

Proposition 7. For each positive integer $n \geq 2$, there exist infinitely many totally real number fields $F$ as in (2.6) of degree $n$.

For the proof, we need: 
LEMMA 8. Let $n \geq 2$ be a positive integer and $\beta \in \mathbb{Z}, \beta \neq 0$. Then there exist infinitely many primes $l$ that satisfy the following two conditions:

(i) $l \nmid \beta n(n-1)$.

(ii) There is some $a(l)$ in $\mathbb{Z}$ such that $\operatorname{ord}_{l}\left(d\left(g_{a}\right)\right)=1$ for all integers $a$ with $a \equiv a(l) \bmod l^{2}$, where we put $g_{a}(X):=X^{n}-a X^{n-1}-\beta$ and denote by $d\left(g_{a}\right)$ the discriminant of $g_{a}(X)$.

Proof. Let $\zeta_{n}$ be a primitive $n$th root of unity, and put

$$
K:=\mathbb{Q}(\sqrt[n]{-\beta(n-1)}) \text { and } N:=K\left(\zeta_{n}\right) .
$$

Since $N / \mathbb{Q}$ is Galois, by the Dirichlet density theorem, there exist infinitely many primes $l$ such that $l \nmid \beta n(n-1)$ and $l$ is completely decomposed in $N$. Take such a prime $l$ and let $\mathcal{L}$ be a prime ideal of $\mathfrak{o}_{K}$ lying above $l$. Since $l$ is a prime element of $\mathcal{L}$, we have

$$
\mathfrak{o}_{K} / \mathcal{L}^{2}=\left\{\left(a_{0}+a_{1} l\right) \bmod \mathcal{L}^{2} \mid a_{0}, a_{1} \in \mathbb{Z} / l \mathbb{Z}\right\} .
$$

Therefore there is some $b$ in $\mathbb{Z}$ such that

$$
b \equiv \sqrt[n]{-\beta(n-1)} \frac{n}{n-1} \bmod \mathcal{L}^{2}
$$

Since $l \nmid \beta n(n-1)$, we have $l \nmid b$. Put $a(l):=b+l$. Let $a$ be an integer with $a \equiv a(l) \bmod l^{2}$ and put $g(X):=X^{n}-a X^{n-1}-\beta$. By Swan [9, Theorem 2], we have

$$
\begin{aligned}
d(g) & =(-1)^{n(n-1) / 2}(-\beta)^{n-2}\left\{(-1)^{n-1}(n-1)^{n-1}(-a)^{n}-n^{n} \beta\right\} \\
& =(-1)^{(n+2)(n-1) / 2} \beta^{n-2}\left\{(n-1)^{n-1} a^{n}+n^{n} \beta\right\} .
\end{aligned}
$$

As the definition of $a$ and (5.1) imply that

$$
a^{n} \equiv a(l)^{n} \equiv b^{n}+n b^{n-1} l \equiv-\beta(n-1)(n /(n-1))^{n}+n b^{n-1} l \bmod l^{2},
$$

we obtain $(n-1)^{n-1} a^{n}+n^{n} \beta \equiv n(n-1)^{n-1} b^{n-1} l \bmod l^{2}$. Hence, (5.2) yields

$$
d(g) \equiv(-1)^{(n+2)(n-1) / 2} \beta^{n-2} n(n-1)^{n-1} b^{n-1} l \bmod l^{2} .
$$

As $l \nmid \beta n(n-1) b$, we have $\operatorname{ord}_{l}(d(g))=1$. This proves our lemma.

Proof of Proposition $\%$. Let $F_{1}, \ldots, F_{t}$ be finitely many distinct fields as in (2.6). It follows from Lemma 8 for $\beta=2$ that there exist some odd prime $l$ and some $a(l)$ in $\mathbb{Z}$ such that $\operatorname{ord}_{l}(d(g))=1$ and $l$ is unramified in each $F_{i}(1 \leq i \leq t)$, where we put $g(X):=X^{n}-a(l) X^{n-1}-2$. Take $n-1$ odd primes $p_{i}(2 \leq i \leq n)$ with $p_{i} \neq l$ satisfying $(2.1)$, and let $a_{1}, \ldots, a_{n}$ be integers which satisfy $(2.2)-(2.5)$,

$$
a_{1} \equiv a(l) \bmod l^{2}, \quad \text { and } \quad a_{i} \equiv 0 \bmod l^{2} \quad \text { for all } i(2 \leq i \leq n) .
$$

Then we define a field $F$ as in (2.6). Since (5.3) implies that $f(X) \equiv$ $g(X) \bmod l^{2}$, we have $\operatorname{ord}_{l}(d(f))=1$. If $d_{F}$ is the absolute discriminant of $F$, then $d(f)=d_{F} \cdot\left(\mathfrak{o}_{F}: \mathbb{Z}[\theta]\right)^{2}$. Hence, $l \mid d_{F}$. Therefore, $l$ is ramified in $F$, and $F \neq F_{1}, \ldots, F_{t}$. 
When the degree $n$ is a power of odd prime, Proposition 9 implies that a field $F$ as in (2.6) is not Galois over $\mathbb{Q}$, because 2 is (totally) ramified in $F$. In particular, when $n=3$, we see that $F$ is not a cyclic cubic field.

Proposition 9. Let $F / \mathbb{Q}$ be a Galois extension of prime power degree, say $l^{t}$. Suppose that $p$ is a prime such that $p \neq l$ and $p \neq \equiv 1 \bmod l$. Then $p$ is unramified in $F$. In particular, if $l$ is odd then 2 is unramified in $F$.

Proof. Let $G:=\operatorname{Gal}(F / \mathbb{Q})$ and $\mathfrak{p}$ a prime ideal of $\mathfrak{o}_{F}$ lying above $p$. For each non-negative integer $m$, we put

$$
G_{m}:=\left\{s \in G \mid s(x) \equiv x \bmod \mathfrak{p}^{m+1} \text { for all } x \text { in } \mathfrak{o}_{F}\right\} .
$$

Then it is known that $\left|G_{0} / G_{1}\right| \mid(N \mathfrak{p}-1)$, and $\left|G_{m} / G_{m+1}\right| \mid N \mathfrak{p}$ for each $m \geq 1$ (cf. Iwasawa [6, Proposition 2.19]), where $N \mathfrak{p}$ is the absolute norm of $\mathfrak{p}$. As $p \neq l$, we obtain $G_{m}=\{1\}$ for all $m \geq 1$. Hence, $\left|G_{0}\right| \mid(N \mathfrak{p}-1)$. Let $f$ be the residue degree of $\mathfrak{p}$ in $F / \mathbb{Q}: N \mathfrak{p}=p^{f}$. Since $F / \mathbb{Q}$ is Galois, both $f$ and $\left|G_{0}\right|$ divide $l^{t}$. By Fermat's little theorem, we obtain $N \mathfrak{p} \equiv p \bmod l$. Then, since the assumption implies that $l \nmid(N \mathfrak{p}-1)$, we have $l \nmid\left|G_{0}\right|$. Hence, $G_{0}=\{1\}$, therefore $p$ is unramified in $F$. This proves our proposition.

\section{References}

[1] C. Batut, K. Belabas, D. Bernardi, H. Cohen and M. Olivier, GP/PARI calculator, Version 2.0.17 (beta).

[2] J. Brinkhuis, Unramified abelian extensions of $C M$-fields and their Galois module structure, Bull. London Math. Soc. 24 (1992), 236-242.

[3] - On the Galois module structure over CM-fields, Manuscripta Math. 75 (1992), 333-347.

[4] L. Childs, The group of unramified Kummer extensions of prime degree, Proc. London Math. Soc. 35 (1977), 407-422.

[5] H. Ichimura, A note on unramified quadratic extensions over algebraic number fields, Proc. Japan Acad. Ser. A 76 (2000), 78-81.

[6] K. Iwasawa, Local Class Field Theory, Oxford Univ. Press, 1986.

[7] F. Kawamoto and Y. Odai, Normal integral bases of $\infty$-ramified abelian extensions of totally real number fields, Abh. Math. Sem. Univ. Hamburg, to appear.

[8] M. Pohst, KANT/KASH calculator, Version 2.2.

[9] R. Swan, Factorization of polynomials over finite fields, Pacific J. Math. 12 (1962), 1099-1106.

Department of Mathematics

Yokohama City University

22-2, Seto, Kanazawa-ku

Yokohama, 236-0027, Japan

E-mail: ichimura@yokohama-cu.ac.jp
Department of Mathematics Faculty of Science, Gakushuin University 1-5-1 Mejiro Toshima-ku Tokyo 171-8588, Japan E-mail: fuminori.kawamoto@gakushuin.ac.jp 